\title{
Acciones educativas dirigidas al personal médico y de enfermería para disminuir las infecciones del tracto sanguíneo relacionadas a catéteres venosos centrales ${ }^{1}$
}

Institución: Hospital Nacional de Niños “Dr. Carlos Sáenz Herrera”

Marlen Villegas Sánchez ${ }^{2}$

Milena Arias Jiménez ${ }^{3}$

Marcela Hernández de Mezerville

\section{CÓMO CITAR}

Villegas, M., Arias, M. y Hernández, M. (2014). Acciones educativas dirigidas al personal médico y de enfermería para disminuir las infecciones del tracto sanguíneo relacionadas a catéteres venosos centrales. Rev. Enfermería Actual en Costa Rica, 27, 1-11. DOI:

http://dx.doi.org/10.15517/revenf.v0i27.16058

\section{RESUMEN}

Introducción. La bacteriemia relacionada a Catéter Venoso Central es una de las principales complicaciones en los pacientes a quienes se les instala un catéter intravascular. Las acciones educativas han mostrado disminución en la tasa de estas infecciones en diferentes estudios. El objetivo fue determinar la tasa de Infecciones del tracto sanguíneo relacionadas al catéter venoso central (ITS/CVC), en el Servicio de Cuidado Intensivo Neonatal, implementar las acciones preventivas y cuantificar el impacto.

Metodología. Es un estudio descriptivo en donde se compara la tasa de ITS/CVC, antes y después de una intervención educativa, en los pacientes ingresados a SECIN. La tasa de ITS/CVC se obtuvo dividiendo el número de éstas entre el número de días-catéter y se multiplicó por 1000.

Resultado. Un resultado relevante es que en el periodo pre intervención se observa una tasa de 32,84 infecciones por 1000 días (cuarto trimestre del 2012 y primer trimestre del 2013) y en el periodo post intervención la tasa fue de 8,28 infecciones por 1000 días. (Tercer trimestre y cuarto trimestre del 2013).

Conclusión. Se concluye que las acciones educativas para disminuir la tasa de ITS/CVC fueron efectivas.

Palabras clave: acción-educativa, bacteriemia, cateterismo, cuidado-intensivo-neonatal, infección-por-catéterintravascular, líneas-intra-arteriales

\footnotetext{
${ }^{1}$ Fecha de recepción: 30 de junio del 2014

Fechas de aceptación: 13 de agosto del 2014

${ }^{2}$ Hospital Nacional de Niños "Dr. Carlos Sáenz Herrera". Costa Rica. Correo electrónico: patitavillegass@gmail.com

${ }^{3}$ Hospital Nacional de Niños “Dr. Carlos Sáenz Herrera”. Costa Rica. Correo electrónico: milenaarias.jimenez@gmail.com

${ }^{4}$ Comité de Prevención y Control de Infecciones. Hospital Nacional de Niños "Dr. Carlos Sáenz Herrera”. Costa Rica. Correo

electrónico: marcelahdm@gmail.com
} 


\title{
Educational initiatives aimed at medical and nursing staff to lower blood tract infections related to central venous catheters ${ }^{1}$
}

Marlen Villegas Sánchez ${ }^{2}$ Milena Arias Jiménez ${ }^{3}$ Marcela Hernández de Mezerville ${ }^{4}$

Institution: National Children's Hospital "Dr. Carlos Sáenz Herrera"

\section{CITED}

Villegas, M., Arias, M. y Hernández, M. (2014). Educational initiatives aimed at medical and nursing staff to lower blood tract infections related to central venous catheters. Rev. Enfermería Actual en Costa Rica, 27, 1-11 . DOI: http://dx.doi.org/10.15517/revenf.v0i27.16058

\begin{abstract}
Introduction. Intravascular catheter-related infection is the major complication in patients with intravascular catheter. Intravascular catheter-related infection rate is derived by dividing the number of these infections between day-line vascular catheters and multiplied $x 1000$. Educational initiatives decrease the rate of intravascular catheter-related infections according to different studies. Objectives: To determine the rate of STI / CVC in the Neonatal Intensive Care Service, implement preventive actions and quantify the impact.

Method. We compared the rate of intravascular catheter-related infections before and after an educational intervention.

Result. In the pre intervention period a rate of 32,84 infections per 1,000 observed days (fourth quarter 2012 and first quarter of 2013) and in the post intervention period the rate is 8,28 infections per 1000 days. (Third quarter and fourth quarter of 2013) infections in 2850 catheter-days and the rate decreased to 5,6, which was statistically significant.
\end{abstract}

Conclusion. Educational initiatives aimed at decreasing the rate of intravascular catheter-related infections were shown to be effective.

Key words: bacteremia, catheterization; intra-arterial-lines; infection, intravascular catheter-related; intensive care-units, neonatal; intensive-care, intervention.

\footnotetext{
${ }^{1}$ Date of reception: June 30, 2014

Fechas de aceptación: August 13, 2014

${ }^{2}$ National Children's Hospital "Dr. Carlos Sáenz Herrera”. E-mail: patitavillegass@gmail.com

${ }^{3}$ National Children's Hospital "Dr. Carlos Sáenz Herrera”. E-mail: milenaarias.jimenez@gmail.com

${ }^{4}$ Committee for Prevention and Control of Infections. National Children's Hospital "Dr. Carlos Sáenz Herrera". E-mail:

marcelahdm@gmail.com
} 


\section{INTRODUCCIÓN}

El uso de los catéteres venosos centrales es una práctica común en las Unidades de Cuidado Intensivo Neonatal (UCIN), para administrar medicamentos, nutrición y monitoreo. Este procedimiento lleva consigo un gran riesgo para el paciente, porque al hacerlo, es necesario lesionar la piel y establecer una comunicación directa con el torrente sanguíneo, que lo expone a la penetración de bacterias en el tejido subcutáneo y en el sistema circulatorio.

Las bacteriemias asociadas con catéteres varían según tipo de catéter, el sitio de inserción, la frecuencia de manipulación del catéter, la severidad de la enfermedad y las condiciones de inserción: electivas o de urgencias.

Hay cuatro rutas reconocidas para la contaminación de los catéteres: 1) la migración de los organismos de la piel en el sitio de inserción en el tracto catéter cutánea y a lo largo de la superficie del catéter con la colonización de la punta del catéter; esta es la ruta más común de infección para los catéteres a corto plazo. 2) la contaminación directa del catéter por el contacto con las manos o los fluidos o dispositivos contaminados. 3) con menor frecuencia, los catéteres pueden llegar a ser colonizados por siembra hematógena desde otro foco de infección. 4) rara vez, la contaminación por infusión podría conducir a infección. (Centro para el Control y Prevención de Enfermedades, CDC, 2011).

Durante cuatro años se realizó en un Hospital de Canadá un estudio de infecciones sanguíneas asociadas al catéter venoso central (CVC) y la tasa de Central Line-Associated Bloodstream Infection (CLABSI) fue de 7,9 por 1000 días de catéter en el inicio del estudio (periodo 1 [agosto 2007-junio 2008]) se redujo gradualmente a lo largo de todo el período de estudio ( $\mathrm{p}=0,034)$ : período 2 (julio de 2008 hasta mayo 2009), 3,3 por 1000 días de catéter; período 3 (junio 2009-abril 2010), 2,6 por 1000 días de catéter, y período 4 (mayo 2010-marzo 2011), 2,2 por 1000 días de catéter. (Ting, Goh \& Osiovich, 2013).

Debido a la relevancia que tienen estos procesos, se ha demostrado que mediante acciones educativas se puede reducir de manera significativa su frecuencia. Se refiere disminución de 4,51 a 2,92 x 1000 días/catéter de infecciones relacionadas a catéter intravascular en seis UCI de adultos. (Sheretz, et al., 2000).

Otros autores reportaron una tasa de 7,8 x 1000 días-catéter preintervención, disminuyéndola a 2.3 y en un estudio de seguimiento a ocho años se disminuyó de 9.7 a 3 x 1000 días-catéter en una población pediátrica. (Bhutta, et al., 2007).

Asimismo, Lisboa y Rello (2008) mencionan que han obtenido una reducción de $40 \%$ en la incidencia de las infecciones relacionadas con el catéter, con una intervención de educación, cuyo objetivo era poner de relieve las medidas basadas en la evidencia asociadas a la prevención de las infecciones asociadas al catéter. Ellos adoptaron medidas educativas, así como la incorporación en la práctica de las medidas basadas en la evidencia, obteniendo resultados parecidos, con una reducción del $21 \%$ en la incidencia de las infecciones asociadas al catéter, pero con variabilidad entre los diferentes centros. El impacto de las medidas de educación en la reducción de la incidencia de infecciones es sostenida por otros trabajos y su importancia en la promoción de la seguridad en los pacientes sugiere la necesidad de su puesta en práctica. 
Recientemente, Pronovost et al. (2006) han evaluado un paquete de medidas destinado a mejorar el cumplimiento de las recomendaciones del CDC acerca de la prevención de la infección relacionada con catéter. La puesta en marcha de las medidas se ha hecho a través de la educación, uso de listas de chequeo (checklist), discusión diaria de las necesidades y de la permanencia de los catéteres, interrupción del procedimiento si no se cumplen las recomendaciones y comunicación de los resultados a los participantes del estudio. Además de obtener una reducción, en los primeros 3 meses, de 2,7 a 0 episodios por cada 1000 catéteres al día, el efecto se mantuvo durante los 18 meses de seguimiento, con una reducción de 7,7 a 1,4 episodios por cada 1000 catéteres al día. La reducción en la incidencia de infecciones relacionadas con catéter, además de reducir los costes, tiene un efecto directo en la seguridad de los pacientes. Estas intervenciones que disminuyen el riesgo de acontecimientos adversos y demuestran un compromiso diario con la seguridad del paciente pueden ser un medio para mejorar los resultados.

Las medidas más importantes incluyen el uso de barreras estériles durante la inserción, el uso de clorhexidina en el punto de inserción, utilización preferente de la vía subclavia frente a femoral y evitar el mantenimiento innecesario del catéter. Pero las medidas cruciales siguen siendo una higiene de manos adecuada, y los principios de control de infección en la prevención. (Saint, Savel, \& Matthay, 2002).

El objetivo de este estudio fue determinar la tasa de Infecciones del tracto sanguíneo de niños y niñas hospitalizadas en el Servicio de Cuidado Intensivo Neonatal (SECIN) del Hospital Nacional de Niños y su relación con el catéter venoso central (ITS/CVC), implementar las acciones preventivas y cuantificar el impacto durante enero a diciembre del 2013.

\section{MATERIALES Y MÉTODO}

Se trata de un estudio de intervención no aleatorizado, descriptivo; donde la población objeto es el total del personal médico y de enfermería del SECIN, así como los pacientes hospitalizados en esta Unidad a los cuales se les coloco Catéter venoso central.

Los instrumentos utilizados para recolectar datos fueron la hoja de Asistencia a charlas, para el control de los participantes.

Para la recolección de los datos de infecciones la VE-01, ficha investigación de infección nosocomial (Y95) y la "Hoja de registro de la vigilancia factores de riesgo infecciones nosocomiales, días exposición". Aplicación del instrumento de la OMS para observaciones de higiene de manos (Formulario de Observación A34) y el formulario de cálculo básico.

Además, se realiza tres veces a la semana recorrido por el SECIN para captar días exposición de CVC y la revisión diaria de los resultados de laboratorio clínico para captar las infecciones intrahospitalarias.

Se realizaron 200 observaciones de higiene de manos pre educación y 200 observaciones post educación.

En la primera etapa del estudio se revisó las tasas de infección relacionada a catéteres venosos centrales, en los pacientes hospitalizados en el Servicio de Cuidado Intensivo Neonatal, "Dr. Carlos Sáenz H" durante el 
período de enero diciembre 2012, de manera retrospectiva y transversal. Se incluyeron todos los pacientes hospitalizados en esta área y con CVC colocado en cualquier sitio anatómico, y que cumplieron con la definición de bacteriemia relacionada a la línea venosa central según las guías del CDC.

En una segunda etapa se implementaron medidas educativas a todo el personal médico y de enfermería y se ejecutaron en conjunto con la Comisión de Catéter Venoso Central. (Véase tabla 2)

\section{Tabla 1}

Servicio de Cuidado Intensivo Neonatal, HNN: Acciones educativas para el personal médico y de enfermería.

\begin{tabular}{clll}
\hline $\begin{array}{l}\text { Tipo de acción } \\
\text { educativa }\end{array}$ & \multicolumn{2}{l}{ Tema } & Personal \\
\hline \multicolumn{1}{c}{ Capacitación } & - & Colocación CVC & Médico \\
& - & Curación y mantenimiento de CVC & Enfermería \\
& & $\begin{array}{l}\text { Protocolo de colocación de sondas urinarias } \\
\text { permanentes }\end{array}$ & \\
& - & Técnica aséptica médica y quirúrgica & \\
& - Higiene de manos & Médico \\
Exposición & $\bullet$ & Bundles $(C V C)$ & Enfermería \\
\hline
\end{tabular}

Fuente: Elaboración propia.

Para calcular la tasa de infecciones sanguíneas asociadas a dispositivos por 1000 catéteres-día, se tomó la fórmula recomendada por el Centro de Control de Enfermedades: Número de ITS asociadas a CVC/Número de catéteres-día x 1000.

Para valorar el impacto de las acciones educativas, se comparan las tasas de infecciones tomando los dos trimestres pre intervención y post intervención. Al igual que observaciones pre y post intervención.

\section{Consideraciones éticas.}

Dentro de las consideraciones éticas, para cada material que se utilizó en el desarrollo de la presente investigación, se respetaron los derechos de autor, incorporando a lo largo del texto las citas bibliográficas correspondientes. Los instrumentos de recolección de datos VE-01 Y-95, hoja de registro de la vigilancia factores de riesgo infecciones nosocomiales, días exposición son los autorizados por la Caja Costarricense de Seguro Social- Componente de Infecciones. Formulario de Observación A34 y el formulario de cálculo básico son los dados por Organización Mundial de la Salud.

En cuanto al análisis de los documentos recolectados (datos), su manejo concuerda con la metodología. 


\section{RESULTADOS}

Según sexo, para el año 2012 no hubo diferencia significativa en la presencia de infecciones asociadas al catéter venoso central, para el 2013 hubo presencia de infecciones en 1,3 masculinos por cada femenino.

Respecto al grupo de edad, obsérvese la tabla 2 en donde se destaca que el grupo de 0 a 30 días es el que presenta mayor número de infecciones $(f=21,40 \%$ para el año 2012 y de igual forma para el año 2013 este grupo es el más vulnerable $\operatorname{con} f=31,66 \%$.

\section{Tabla 2}

Servicio de Cuidado Intensivo Neonatal, HNN: Infecciones del torrente sanguíneo asociadas a catéter venoso central según grupo de edad, 2012-2013.

\begin{tabular}{crc}
\hline & $\mathbf{2 0 1 2}$ & $\mathbf{2 0 1 3}$ \\
\hline Grupo de edad & Frecuencia & Frecuencia \\
0 a 30 días & 21 & 31 \\
31 a 60 días & 7 & 10 \\
61 a 90 días & 15 & 3 \\
>91 días & 10 & 3 \\
Total & 53 & 47 \\
\hline
\end{tabular}

Fuente: Estadísticas de Servicio de Cuidado Intensivo Neonatal, CCPIN, 2012-2013

Según tipo de catéter colocado en el Servicio de Cuidado Intensivo Neonatal (CCPIN); el catéter central intravascular percutáneo (PICC) se colocó con más frecuencia. En el período de enero-diciembre 2012 se colocó a 477 pacientes con 4561 días dispositivo; se presentó 53 infecciones para una tasa anual de 11,62. Por otro lado, en enero-diciembre del 2013 fueron colocados 476 catéteres con 5681 días-dispositivo, se presentó 47 infecciones para una tasa anual de 8,27. 


\section{Tabla 3}

Servicio de Cuidado Intensivo Neonatal, HNN: Porcentaje de gérmenes aislados en infecciones del torrente sanguíneo asociadas a CVC, SECIN- HNN

\begin{tabular}{lcccc}
\hline & $\mathbf{2 0 1 2}$ & & $\mathbf{2 0 1 3}$ \\
\hline GERMENES & FREQUENCIA & $\mathbf{\%}$ & FREQUENCIA & $\mathbf{\%}$ \\
C. freundii & - & - & 1 & 2 \\
C. galbrota & - & - & 1 & 2 \\
S. mitis & - & - & 1 & 2 \\
A.junnii & 1 & 2 & - & - \\
K.oxytoca & 2 & 4 & 1 & 2 \\
P.aeruginosa & 1 & 2 & 1 & 2 \\
P. putida & 1 & 2 & - & \\
S. coagulasa negativo & - & - & 2 & 4 \\
S.marcescens & 8 & 15 & 2 & 4 \\
E.cloacae & 3 & 5 & 4 & 7,54 \\
P. mirabilis & 1 & 2 & - & \\
E. faecalis & 8 & 15 & 4 & 7,54 \\
SAMR & 3 & 5 & 4 & 7.54 \\
K.pneumoniae & 13 & 24 & 5 & 9,40 \\
E.coli & 6 & 10 & 5 & 9,40 \\
C.parapsillosis & 3 & 5 & 6 & 11 \\
C.albicans & 2 & 4 & - & \\
C. tropicalis & 1 & 2 & - & \\
S.epidermidis & 9 & 16 & 16 & 30 \\
S. saprophyticus & 1 & 2 & - & \\
S. warnerii & 1 & 2 & - & \\
S. haemolythicus & 1 & 2 & - & \\
\hline \multicolumn{1}{c}{ Total } & 55 & 100 & 53 & 100 \\
\hline Estadicas & & & \\
\hline
\end{tabular}

Fuente: Estadísticas de Servicio de Cuidado Intensivo Neonatal, CCPIN, 2012-2013

El germen aislado más frecuente en los casos de infecciones del torrente sanguíneo asociado a CVC como se observa en la tabla 3 fue el $S$. epidermidis en ambos períodos. Las bacterias gram negativas ocuparon el segundo lugar. 


\section{Gráfico 1}

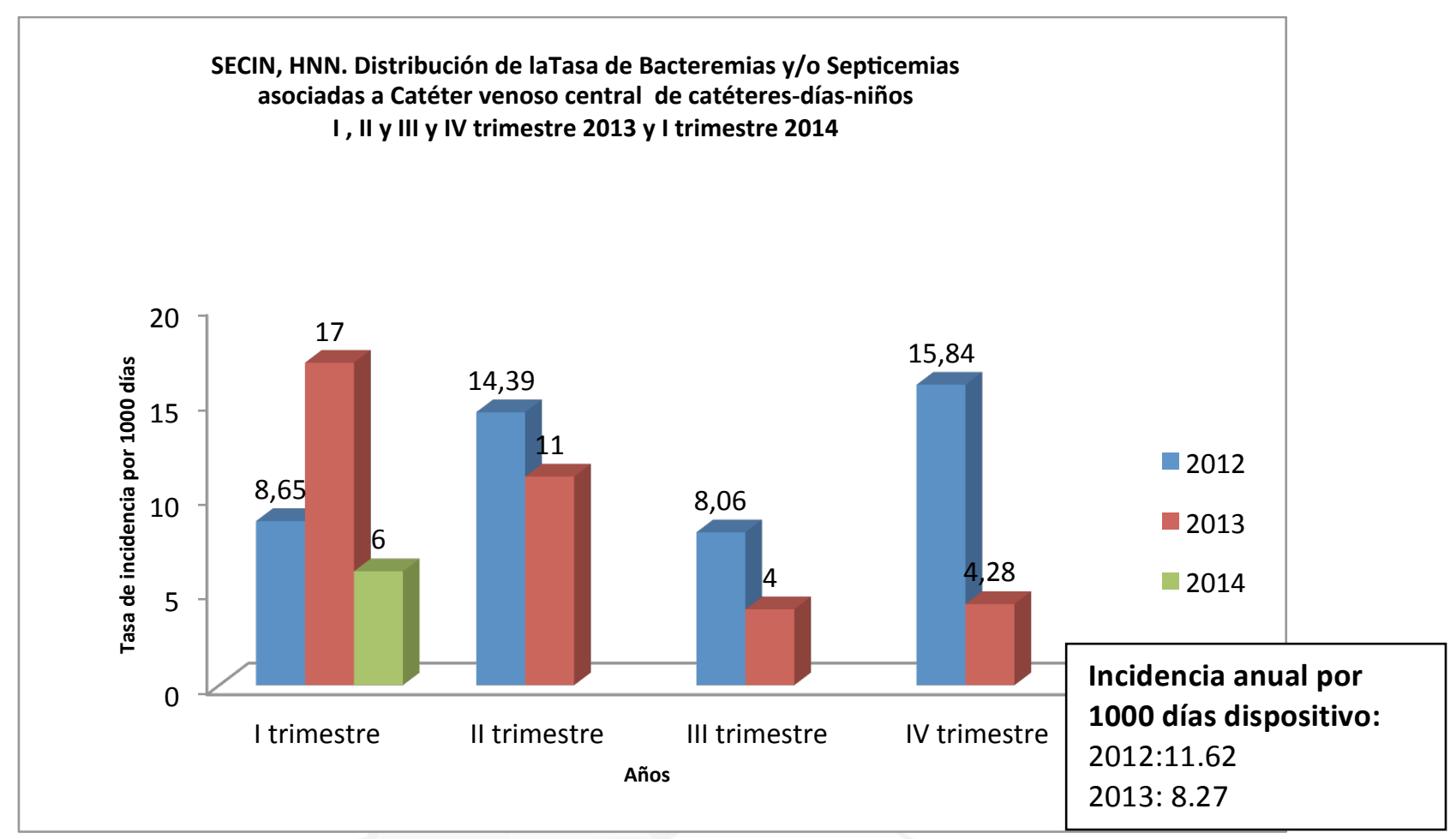

Fuente: Estadísticas de Servicio de Cuidado Intensivo Neonatal, CCPIN, 2012-2013 y 2014

Como se observa en el grafico 1, fue a partir del segundo semestre del 2013 donde se inició la capacitación y se comienza a observar un descenso importante de la tasa de infecciones.

Igualmente, en el recuadro se puede observar las tasas de incidencia anuales en donde se observa una disminución de 3.35 infecciones por 1000 días-dispositivo entre el 2012 y 2013.

En el periodo pre-intervención se observa una tasa de 32,84 infecciones por 1000 días (cuarto trimestre del 2012 y primer trimestre del 2013) y en el periodo post intervención la tasa es de 8, 28 infecciones por 1000 días. (Tercer trimestre y cuarto trimestre del 2013).

En cuanto a las observaciones de higiene de manos, en el período pre intervención el porcentaje de cumplimiento fue de $63 \%$, post intervención $77 \%$.

Se capacitó al 71\% del personal de enfermería y 86\% del personal médico. 


\section{DISCUSIÓN}

Este estudio demostró la eficacia de un programa educativo para mejorar el nivel de conocimientos, actitudes y prácticas del personal hospitalario aunque no se haya logrado capacitar el $100 \%$ de la población.

La capacitación de los funcionarios de salud garantiza la eficiencia de los programas de prevención y control de infecciones, sin embargo, a pesar de brindarse la capacitación no se garantiza la adherencia a las medidas de prevención en un $100 \%$.

Varios estudios han demostrado la eficiencia de la educación, como en un estudio en el cual, al inicio de la capacitación, el cumplimiento de la técnica del lavado de manos fue del $7 \%$, elevándose al $78 \%(\mathrm{p}<0,05)$ transcurridos de tres a seis meses después de la capacitación. (Huaroto, et al., 2013).

La adherencia por parte de los trabajadores de la salud a los procedimientos de higiene de manos recomendados han sido proporcionados como variables, cuyos índices de línea de base media oscilan entre 5\% y $89 \%$ y un promedio total de $38,7 \%$. El desempeño relacionado con la higiene de manos varía según la intensidad del trabajo y otros factores varios (OMS, 2009).

Posterior a la capacitación en temas tales como técnica aséptica médica y quirúrgica, manipulación y curación de catéter venoso central, se ha demostrado importante disminución en las tasas de infección.

En el estudio pre-intervención, la frecuencia de infecciones del tracto sanguíneo asociadas a CVC fue de 11,62 x 1000 días-catéter, valor que consideramos elevado con base en otros estudios. Se aplicaron las acciones educativas y se cuantificó la efectividad demostrada con una reducción de 71\% (3,35 x 1000 días-catéter). Los resultados son similares a lo reportado en estudios previos, siendo el más representativo el de Costello, Forbes, Graham, Potter-Byone, Sandora \& Laussen. (2008), que redujeron una tasa de 7,8 x 1000 días-catéter a 2,3.

En un estudio en Alemania ponen de manifiesto la importancia de la normalización y la coherencia de los procedimientos médicos y de higiene en las UCIN. Además, abogan por un alto nivel de comunicación y cooperación entre el personal de la NICU y campos relacionados. (Leistner, Thürnagel, Schwab, Piening, Gastmeier \& Geffers., 2013).

La tasa mediana de CLABSI es de 7,9 por 1000 días-catéter en el inicio del estudio (periodo 1 [agosto 2007-junio 2008]) se redujo gradualmente a lo largo de todo el período de estudio $(\mathrm{p}=0,034)$ : período de 2 (julio de 2008 hasta mayo 2009), 3,3 por 1.000 días de catéter; período de 3 (junio 2009 a abril 2010), 2,6 por 1.000 días de catéter; y el período de 4 (desde mayo 2010 hasta marzo 2011), 2,2 por 1.000 días de catéter. (Ting, Goh \& Osiovich., 2013)

La mayoría de las ITS fueron provocadas por Staphylococcus coagulasa (-) y cerca de una quinta parte por Cándida albicans. Estudios refieren que en neonatos y niños de todas las edades los gérmenes más frecuentes asociados a la ITS son Staphylococcus coagulasa (-) 69\%, y hasta un 93\% de las especies son meticillin resistentes (SMR); otros Gram (+) como S. aureus y Enterococcus (9\%), bacilos Gram negativos (19\%) con 
predominio de Enterobacter, Pseudomonas, Klebsiella, Serratia marcescens, y Candida spp (3\%). (Londoño, Ardilia y Ossa, 2011).

El perfil microbiológico predominante fue por contaminantes comunes de la piel y gérmenes hospitalarios Gram negativos con altos índices de resistencia a antibióticos. (Londoño, et al. 2011).

\section{CONCLUSIONES}

En conclusión, se demostró que las acciones educativas reducen la incidencia de infecciones del tracto sanguíneo asociadas a CVC, siendo nuestro objetivo continuar con las capacitaciones, observaciones e implementar el cumplimiento de los Bundles para reducir aún más las infecciones.

\section{Agradecimiento}

Al personal de enfermería y médico por el gran trabajo y esfuerzos que realizaron en disminuir las infecciones en el Servicio de Cuidado Intensivo Neonatal.

\section{REFERENCIAS BIBLIOGRÁFICAS}

Bhutta, A., Gilliam, C., Honeycut, M., Schexnayder, S., Green, J., Moss, M. \& Anand, K.J. (2007). Reduction of bloodstream infections associated with catheters in pediatric intensive care unit: Stepwise approach. BMJ, 334(7589), 362-5.

Centro para el Control y Prevención de Enfermedades (CDC). (2011). Guidelines for the Prevention of Intravascular Catheter-Related Infections. Traducción y notas por Fabiana Ciccioli y Jose Luis do Pico. En: www.cdc.go.cr

Costello, J.M., Forbes, M.D., Graham, D.A., Potter-Byone, G., Sandora, T.J. \& Laussen P.C. (2008). Systematic intervention to reduce central line-associated bloodstream infection rates in a pediatric intensive care unit. Pediatrics, 121, 915-23.

Huaroto, L.M., Lam, C., Mucha, R., Chávez, J., Tanta, J., Alvarezcanok, J. y Ticona, E. (2013). Impacto de un programa de capacitación para la prevención de infecciones intrahospitalarias en un hospital general. Trauma Fund MAPFRE, 24 (2), 126-131.

Leistner, R., Thürnagel, S., Schwab, F., Piening, B., Gastmeier, P., \& Geffers, C. (2013). The impact of staffing on central venous catheter-associated bloodstream infections in preterm neonates - results of nation-wide cohort study in Germany. Antimicrob Resist Infect Control, 2, 11.

Lisboa, T. y Rello, J. (2008). Prevención de infecciones nosocomiales: estrategias para mejorar la seguridad de los pacientes en la Unidad de Cuidados Intensivos. Med. Intensiva, 32 (5). 
Londoño, A.L., Ardila, M. y Ossa, D. (2011). Epidemiología de la infección asociada a catéter venoso central. Rev Chil Pediatr, 82(6), 493-501 Recuperado de: http://www.scielo.cl/scielo.php?pid=S0370$41062011000600003 \&$ script $=$ sci_arttext

Organización Mundial de la Salud (OMS). (2009). Guía de la OMS sobre Higiene de Manos en la Atención de la Salud. Recuperado de: http://www.med.unlp.edu.ar/archivos/noticias/guia_lavado_de_manos.pdf

Pronovost, P., Needham, D., Berenholtz, S., Sinopoli, D., Chu H, Cosgroves S, Sexton, B., Hyzy, R. Welsh, R. Roth, G., Bander, J., Kepros, J. \& Goeschel, C. (2006). An intervention to decrease catheter-related bloodstream infections in the ICU. The New England Journal of Medicine, 355(26), 2725-32.

Saint, S., Savel, R.H. \& Matthay, M.A. (2002). Enhancing the safety of critically ill patients by reducing urinary and central venous catheter related infections. American Journal of Respiratory and Critical Care Medicine, 165(11), 1475-1479. doi: $10.1164 / \mathrm{rccm} .2110035$.

Sherertz, R.J., Ely, E.W., Westbrook, D.M., Gledhill, K.S., Streed, S.A., Kiger, B., Flynn, L., Hayes, S., Strong, S., Cruz, J., Bowton, D.L., Hulgan, T. \& Haponik, E. (2000). Education of Physicians-in-Training Can Decrease the Risk for Vascular Catheter Infection. Ann Intern Med. 132(8), 641-648. doi:10.7326/0003-4819-132-8200004180-00007.

Ting, J.Y., Goh, V.S. \& Osiovich H. (2013). Reduction of central line-associated bloodstream infection rates in a neonatal intensive care unit after implementation of a multidisciplinary evidence-based quality improvement collaborative: A four-year surveillance. Journal Canadien des Maladies Infectieuses et de la Microbiologie Medicale / AMMI Canada, 24(4), 185-190. 